\title{
Measurement and laser control of attosecond charge migration in ionized iodoacetylene
}

\section{Journal Article}

\section{Author(s):}

Kraus, Peter M.; Mignolet, Benoît; Baykusheva, Denitsa; Rupenyan-Vasileva, Alisa Bohos (iD; Horný, Lubos; Penka, Emmanuel F.; Grassi, Guido; Tolstikhin, Oleg I.; Schneider, Johannes; Jensen, Frank; Madsen, Lars B.; Bandrauk, André D.; Remacle, Françoise; Wörner, Hans Jakob

\section{Publication date:}

2015-11-13

Permanent link:

https://doi.org/10.3929/ethz-a-010782069

\section{Rights / license:}

In Copyright - Non-Commercial Use Permitted

\section{Originally published in:}

Science 350(6262), https://doi.org/10.1126/science.aab2160 


\title{
Measurement and laser control of attosecond charge migration in ionized iodoacetylene
}

\author{
P. M. Kraus ${ }^{1}$, B. Mignolet ${ }^{2,3}$, D. Baykusheva ${ }^{1}$, A. Rupenyan ${ }^{1}$, L. Horný ${ }^{1}$, \\ E. F. Penka ${ }^{4}$, G. Grassi ${ }^{1}$, O. I. Tolstikhin ${ }^{5}$, J. Schneider ${ }^{1}$, F. Jensen ${ }^{6}$, \\ L. B. Madsen ${ }^{7}$, A. D. Bandrauk ${ }^{4}$, F. Remacle ${ }^{2}$, and H. J. Wörner ${ }^{1 *}$ \\ ${ }^{1}$ Laboratorium für Physikalische Chemie, ETH Zürich, 8093 Zürich, Switzerland \\ ${ }^{2}$ Department of Chemistry, B6c, Université de Liège, B4000 Liège, Belgium \\ ${ }^{3}$ PULSE Institute and Department of Chemistry, Stanford University, Stanford, \\ California 94305, United States \\ ${ }^{4}$ Laboratoire de Chimie Théorique, Université de Sherbrooke, Quebec, Canada, J1K 2R1 \\ ${ }^{5}$ Moscow Institute of Physics and Technology, Dolgoprudny 141700, Russia \\ ${ }^{6}$ Department of Chemistry, Aarhus University, 8000 Aarhus C, Denmark \\ ${ }^{7}$ Department of Physics and Astronomy, Aarhus University, 8000 Aarhus C, Denmark \\ *Corresponding author: hwoerner@ethz.ch; www.atto.ethz.ch.
}

The ultrafast motion of electrons and holes following light-matter interaction is fundamental to a broad range of chemical and biophysical processes. Here we advance high-harmonic spectroscopy to resolve spatially and temporally the migration of an electron hole immediately following ionization of iodoacetylene, while simultaneously demonstrating extensive control over the process. A multidimensional approach, based on the measurement of both even and odd harmonic orders, enables us to reconstruct both quantum amplitudes and phases of the electronic states with a resolution of $\sim 100$ attoseconds. We separately reconstruct quasi-field-free and laser-controlled charge migration as a function of the spatial orientation of the molecule and determine the shape of the hole created by ionization. Our technique opens the prospect of laser control over electronic primary processes. 
Ultrafast charge transfer plays a key role in chemical reactions, biological processes and technical applications. For example, charge transfer after photoexcitation on the femtosecond time scale and the associated long-lived coherences observed in photosynthetic systems (1) or photovoltaic blends (2) have been invoked to explain the high energy-conversion efficiencies of these systems. These charge transfer phenomena are driven by nuclear motion, but typically involve a much faster purely electronic response. This phenomenon was predicted theoretically and called charge migration to distinguish it from the nuclear-dynamics-driven charge transfer (3-6). Charge migration arises whenever multiple electronic states are coherently populated. Charge migration is likely to be responsible for the unexpected selectivity in photofragmentation of ionized peptides (7), leading to the concept of charge-directed chemistry (8). Moreover, it offers approaches to probing electron-correlation and orbital-relaxation phenomena $(3,5,6)$. These prospects make the measurement of charge migration a key goal of attosecond science (9). Even more promising is the prospect of controlling charge migration. Steering electrons inside molecules is expected to yield control over reactivity, to provide access to regions of potential-energy surfaces that usually remain unexplored and thereby to open novel reaction pathways (10).

Previously described techniques for measuring electronic dynamics include transient absorption (11), sequential double ionization (12) and photofragmentation spectroscopy (13). Although pioneering work on phenylalanine recently reported a resolution of better than 4.5 fs (13), such measurements of intramolecular dynamics are inherently limited in temporal resolution by the use of an infrared pulse. Electronic dynamics following strong-field ionization (SFI) have also been inferred from high-harmonic spectra. This approach has been established in seminal studies $(14,15)$, but a direct reconstruction of the dynamics has remained out of reach, either because no reconstruction approach was established or due to a lack of experimental data. Another pioneering approach demonstrated the tomographic reconstruction of time-dependent orbitals at the expense of temporal resolution (16).

Here we advance high-harmonic spectroscopy (HHS) to reconstruct the full quantum dynamics of charge migration in spatially-oriented polar molecules with $\sim 100$ attosecond resolu- 
tion and to demonstrate extensive control over the process. We measure both amplitudes and phases of high-harmonic emission from oriented molecules at multiple wavelengths of the driving field. Scaling the wavelength is equivalent to tuning the excursion time of the photoelectron wave packet on the attosecond time scale (17). We show that this set of experimental data is sufficient to reconstruct the amplitudes and phases of the transiently occupied electronic states of the cation and to determine the initial shape of the hole created by SFI.

We demonstrate our technique on iodoacetylene (HCCI), because it encompasses several general characteristics of polar polyatomic molecules. These include (i) the simultaneous population of multiple electronic states of the cation by SFI, (ii) strong laser-induced coupling between these states and (iii) a strong alignment dependence of this coupling. An energy-level diagram of $\mathrm{HCCI}^{+}$is shown in Fig. 1. The $\tilde{\mathrm{X}}^{+2} \Pi$ ground state and $\tilde{\mathrm{A}}^{+2} \Pi$ first excited state (with vertical ionization potentials $I_{\mathrm{p}}^{\tilde{\mathrm{X}}^{+}}=9.71 \mathrm{eV}$ and $I_{\mathrm{p}}^{\tilde{\mathrm{A}}^{+}}=11.94 \mathrm{eV}(18)$ ) are both appreciably populated by strong-field ionization (supplementary material (SM) section 4) and are coupled by a large transition dipole moment (1.35 atomic units, which is equivalent to 3.43 debye) lying parallel to the molecular axis. This large value originates from the charge-transfer nature of this $\pi \rightarrow \pi^{*}$ transition (19).

We use control over the spatial orientation of the molecule to separately reconstruct quasifield-free and laser-driven charge migration. For molecules aligned perpendicular to the laser polarization (Fig. 1b), the effect of the laser field on charge migration is negligible, as we discuss below. For parallel molecules (Fig. 1c), the laser field induces substantial population transfer between the $\tilde{\mathrm{X}}^{+}$and $\tilde{\mathrm{A}}^{+}$states which depends on the head-to-tail orientation of the molecule.

The concept of the experiment is presented in Fig. 2a. Control over the spatial orientation of the molecules is achieved by impulsively orienting the molecules (20). The fixed-in-space ensemble of molecules is then interrogated by a high-harmonic generation (HHG) pulse one rotational period (157.0 ps) later under otherwise field-free conditions. The time resolution in our experiment arises from the sub-cycle nature of the HHG process: Every emitted harmonic order can be associated with a unique transit time of the electron wave packet in the continuum 


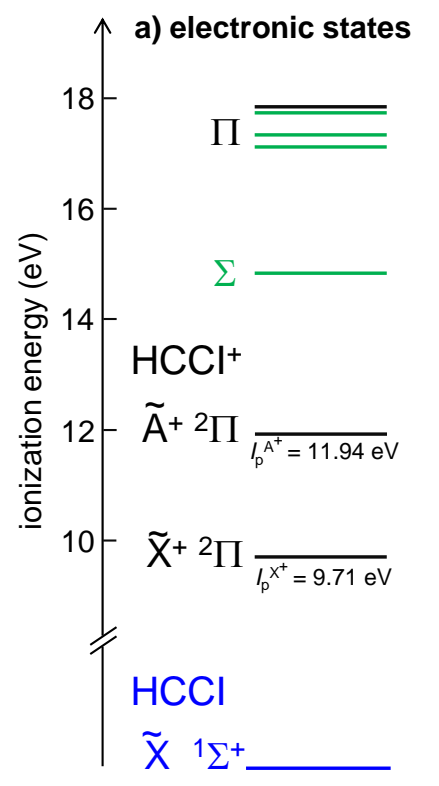

b) quasi-field-free charge migration

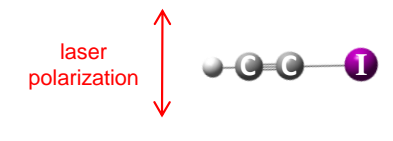

hole density: $\left|\left\langle\Phi^{N-1}(t) \mid \Phi^{N}\right\rangle\right|^{2}$

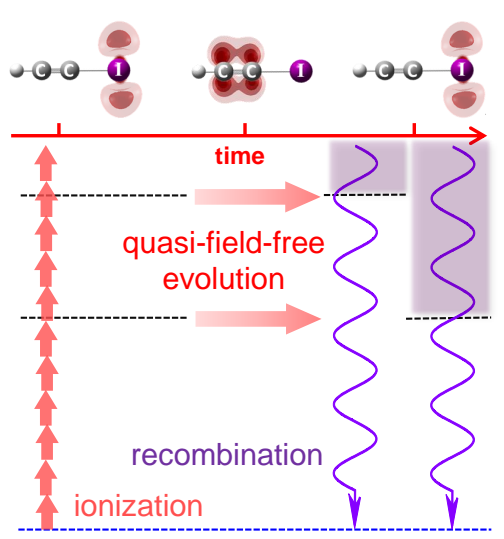

c) laser-controlled charge migration

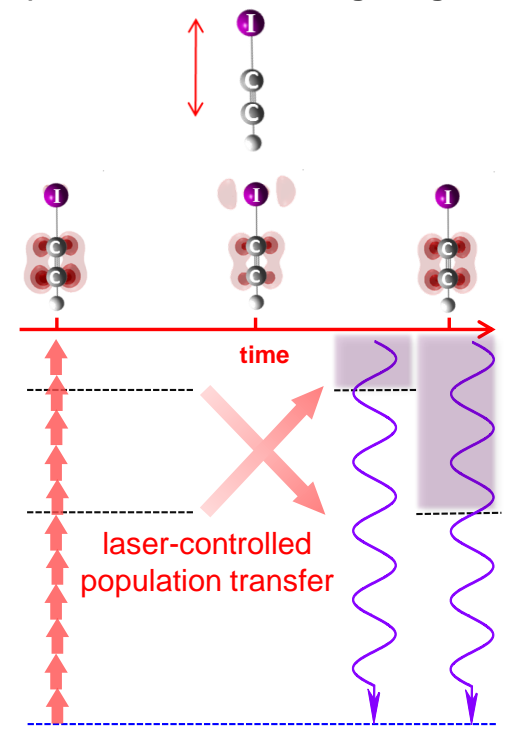

Figure 1: Electronic energy levels and orientation dependence of charge migration in the iodoacetylene cation. (a) Energy-level diagram and illustration of (b) quasi-field-free and (c) laser-controlled charge migration. The energies are computed at the EOM-IP-CCSD/cc-pVTZ level of theory. Strong-field ionization by infrared photons (red arrows) prepares the electron hole. Its evolution is encoded in the high-harmonic emission (violet arrows) at the instant of recombination. For molecules aligned perpendicular to the laser field as shown in (b), the populations of the $\tilde{\mathrm{X}}^{+}$and $\tilde{\mathrm{A}}^{+}$states are time-independent. Charge migration takes place as under field-free conditions. The laser field induces strong population transfer between the $\tilde{\mathrm{X}}^{+}$ and $\tilde{\mathrm{A}}^{+}$states for molecules aligned parallel to the laser field as depicted in (c). The evolution of the hole density is represented by $\left|\left\langle\Phi^{N-1}(t) \mid \Phi^{N}\right\rangle\right|^{2}$, where $\Phi^{N}$ and $\Phi^{N-1}(t)$ are the $N$ and $N-1$ electron wave functions of the neutral and ionized molecule, respectively. 
a) orientation / alignment pulse

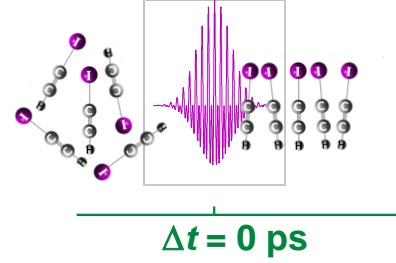

\section{HHG pulse}
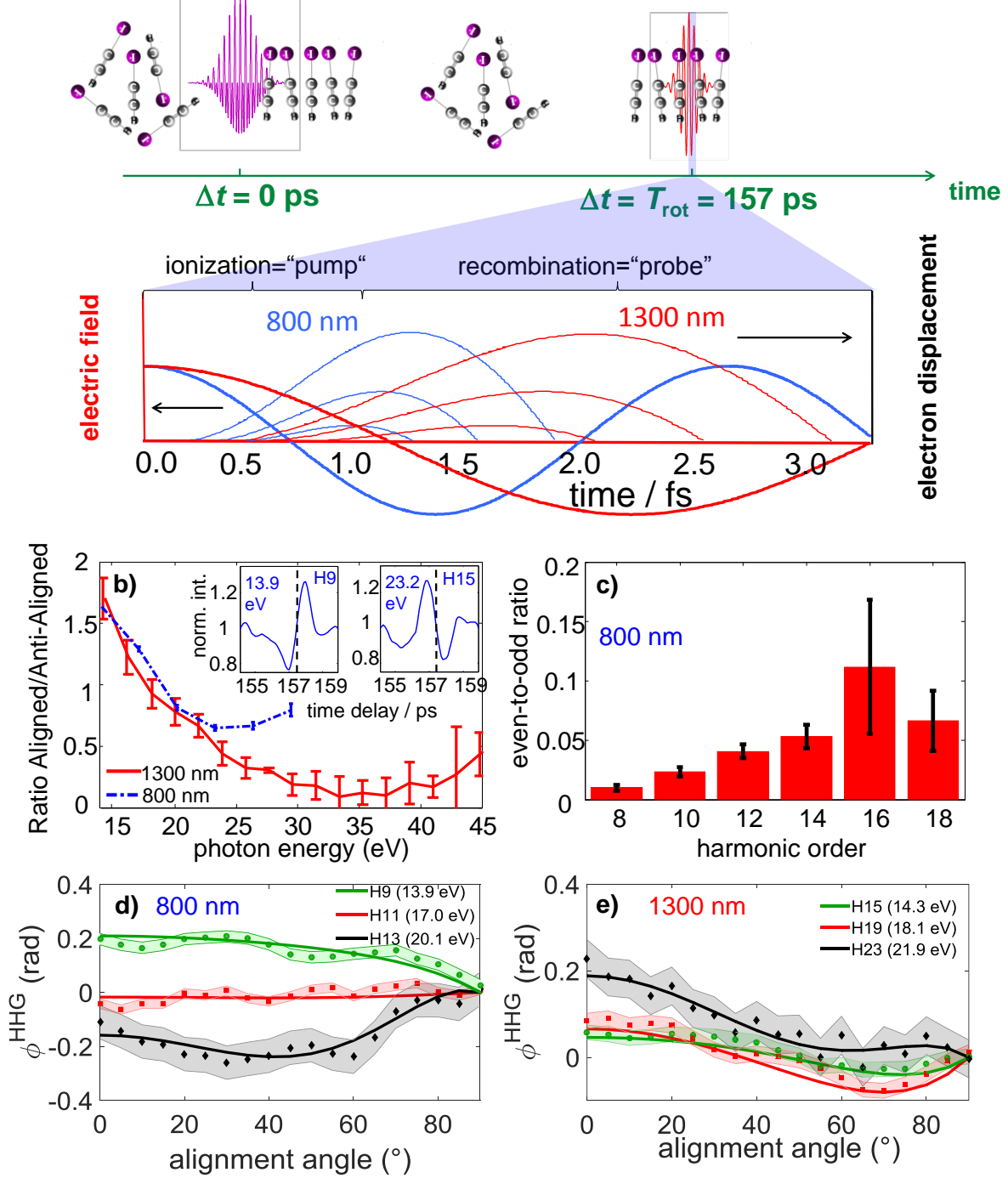

Figure 2: HHG monitoring of charge migration. (a) Concept of the experiment: HHG (upper panel) provides a unique transit-time-to-energy mapping as a consequence of the subcycle timing of the electron trajectories (thin lines, lower panel) in the laser field (thick lines). (b) Intensity ratio of high harmonics emitted from molecules aligned parallel or perpendicular to the laser field for $800 \mathrm{~nm}\left((1.1 \pm 0.2) \cdot 10^{14} \mathrm{~W} / \mathrm{cm}^{2}\right)$ and $1300 \mathrm{~nm}\left((0.9 \pm 0.1) \cdot 10^{14} \mathrm{~W} / \mathrm{cm}^{2}\right)$. The insets show the variation of the intensities of selected harmonic orders of $800 \mathrm{~nm}$. (c) Even-toodd ratio for oriented molecules $(800 \mathrm{~nm})$. (d,e) Alignment dependence of the high-harmonic phase $\phi^{\mathrm{HHG}}$ using $800 \mathrm{~nm}$ and $1300 \mathrm{~nm}$ pulses, respectively. A sum of the four lowest evenorder Legendre polynomials was fitted to the experimental data. The error bars (shaded areas) in all figures correspond to the standard deviation of the measured signal fluctuations. All data were obtained by averaging the signals from at least 1500 laser shots. 
by experimentally selecting the short electron trajectories $(21,22)$.

The reconstruction of charge migration in the cation requires several observables. The ratio of emission intensity between molecules aligned parallel or perpendicular to the polarization of the probe pulse are shown in Fig. $2 b$. These ratios exhibit a minimum located at markedly different photon energies: $23.2 \mathrm{eV}$ for $800 \mathrm{~nm}$ (2.67 fs laser-field period) and $35.3 \mathrm{eV}$ for $1300 \mathrm{~nm}$ (4.33 fs laser-field period). Such a strong wavelength dependence of the observed minimum cannot be contained in the photorecombination matrix elements and indicates attosecond electronic dynamics in the cation $(23,24)$. Purely structural minima contained in the photorecombination matrix elements do not shift in photon energy when the driving wavelength is changed (25).

Spatially resolving charge migration in the general class of polar molecules additionally requires orientation (i.e. a head-to-tail order) resulting in even-harmonic emission. This is achieved using one phase-controlled two-color $(800+400 \mathrm{~nm})$ laser pulse for impulsive orientation (26). Figure $2 \mathrm{c}$ shows the intensity ratio of the even harmonics to the averaged intensity of the two adjacent odd harmonic orders (the even-to-odd ratio) observed using a HHG driving wavelength of $800 \mathrm{~nm}$.

A full characterization of electronic quantum dynamics becomes possible through the additional measurement of the phase of high-harmonic emission. We measure the phase $\phi^{\mathrm{HHG}}$ as a function of the alignment angle of the molecules by generating high harmonics in two spatially separated sources in the gas jet (27). Here, we extend this technique to a broad range of wavelengths by working with a liquid-crystal mask that introduces a voltage-tunable phase shift in one half of the unfocussed probe-beam wave front (SM section 1.3). Phase measurements for driving wavelengths of $800 \mathrm{~nm}$ and $1300 \mathrm{~nm}$ are shown in Figs. 2d and 2e for selected harmonic orders. The phase variation as a function of the alignment angle strongly depends on the harmonic order and driving wavelength, not just the emitted photon energy, again suggesting a pronounced charge migration on the attosecond time scale. The phase differences for molecules aligned parallel and perpendicular to the polarization of the laser field for all harmonic orders are summarized in the SM, section 1.3. 
The reconstruction of charge migration in terms of initial (as prepared by ionization at $t^{\prime}$ ) and final (at the instant of photorecombination, $t$ ) populations and phases of the states of the cation is a numerical inversion problem employing a generalized theory of HHG (SM, section 2) which describes all experimental intensity ratios and phase differences between molecules aligned parallel or perpendicular to the laser field. The initial and final populations and phases are retrieved in a global nonlinear least-squares optimization using a Levenberg-Marquardt algorithm with multiple starting values. Our theory includes all relevant electronic states, the continuum structure through the use of scattering-wave matrix elements (28), nuclear motion through autocorrelation functions derived from photoelectron spectra (SM section 2.3.1) and the molecular axis distribution. The photorecombination dipole matrix elements and the angular variation of the ionization rates are calculated theoretically. These quantities are experimentally accessible using narrow-band extreme-ultraviolet sources and/or charged-particle detection, which were not available in this study. Because the emission from several channels adds coherently, our experiment is sensitive to both the amplitudes and the phases of the involved electronic states. The mapping from photon energy to transit time $\left(\tau=t-t^{\prime}\right)$ is performed using quantum electron trajectories obtained by the saddle-point method (29-31).

We first reconstruct charge migration for molecules aligned perpendicular to the $800 \mathrm{~nm}$ driving field. The reconstructed initial populations are given in Fig. 3b. These results compare well, both with time-dependent density functional theory (TDDFT, SM section 4 (32)) and with weak-field asymptotic theory (WFAT, SM section 2.3.3 (33)). Combined with the experimentally retrieved initial phase $\Delta \varphi=\varphi_{\tilde{\mathrm{A}}^{+}}(\tau=0)-\varphi_{\tilde{\mathrm{X}}^{+}}(\tau=0)$ (Fig. 3c) between the ground and first excited electronic state of the cation, charge migration is reconstructed as shown in Fig. 3a. The spatial representation of the electron densities further requires the computation of molecular orbitals, which is done at the Hartree-Fock level of theory to be consistent with the calculation of the photorecombination matrix elements. It also requires the knowledge of the difference of the vertical ionization potentials of the field-free eigenstates, which determines the oscillation period of 1.85 fs and is known from photoelectron spectroscopy (18). Strong-field ionization is found to create a one-electron hole localized at the iodine-side of the molecule 
(Fig. 3d), compatible with the low ionization potential and high polarizability of the I atom. Subsequently, the hole delocalizes over the molecule and then localizes at the acetylene-side after 930 as. Because the differential Stark shift of the $\tilde{\mathrm{X}}^{+}$and $\tilde{\mathrm{A}}^{+}$levels at the maximal applied field amounts to only $18 \mathrm{meV}$ (SM section 3, Fig. S11) or $0.8 \%$ of the energy-level separation, and population transfer is absent by symmetry, the reconstructed dynamics correspond to quasi-field-free charge migration.

We find a $\sim \pi$ difference in the relative initial phase of the $\tilde{\mathrm{X}}^{+}$and $\tilde{\mathrm{A}}^{+}$states for parallel alignment (Fig. 3c), which implies that the hole is created on the acetylene side (Fig. 3e). This means that the electron hole is created on the opposite side from where the electron tunneled because ionization via the iodine atom is dominant (SM Fig. S5). The reconstructed hole is moreover consistent with ionization to the lowest-lying multi-electron eigenstate of the cation in a static field (see SM Fig. S12).

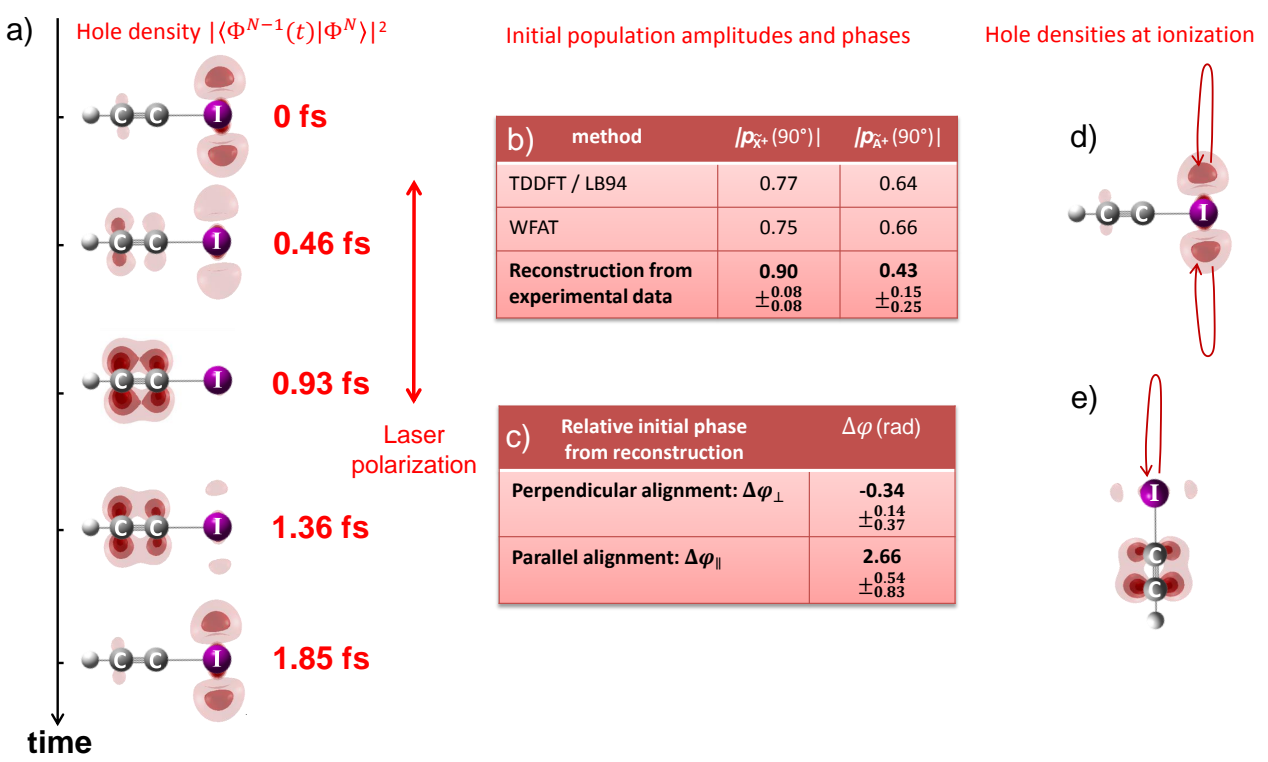

Figure 3: Reconstruction of quasi-field-free charge migration. (a) The reconstructed electron dynamics are displayed as a function of time after ionization. Reconstructed values are given for (b) the population amplitudes $\left|p_{i}\right|\left(i=\tilde{\mathrm{X}}^{+}, \tilde{\mathrm{A}}^{+}\right)$for perpendicular alignment, which are compared to theory (details see text), and (c) the relative initial phase $\Delta \varphi$. All error bars in this work were determined from the experimental errors and the uncertainties in the intensity and alignment axis distribution (SM section 2.4). The reconstructed hole density at the time of ionization is shown for (d) perpendicular and (e) parallel alignment. The arrows schematically illustrate the direction of excursion of the continuum electron wave packet. 
The subsequent charge migration for parallel alignment is controlled by the laser field (Fig. 4) and differs substantially from the field-free evolution (Fig. 3a). We first concentrate on the results obtained with $800 \mathrm{~nm}$ pulses. Figure 4 a shows the fractional population of the $\tilde{\mathrm{X}}^{+}$ ground state (solid blue line) and the relative phase $\Delta \varphi(\tau)=\varphi_{\tilde{\mathrm{A}}^{+}}(\tau)-\varphi_{\tilde{\mathrm{X}}^{+}}(\tau)$ between the $\tilde{\mathrm{X}}^{+}$and $\tilde{\mathrm{A}}^{+}$states (dashed cyan line) for all reconstructed delays. The first striking feature is the strong depletion of the $\tilde{\mathrm{X}}^{+}$state for early transit times. The population of the $\tilde{\mathrm{X}}^{+}$state reaches a minimum at $1.02 \mathrm{fs}$ before increasing again. The relative phase between the two eigenstates shows a pronounced jump around the time of maximal depopulation of the ground state. Figure 4a also shows the reconstructed hole density at selected transit times for electron tunneling via the iodine atom. For electron tunneling via the hydrogen atom (Fig. 4b) the population transfer is markedly different than for tunneling via iodine (Fig. 4a). Again, the ground state is strongly depopulated at the first reconstructed delay, but its repopulation begins earlier than in Fig. 4a. One of the crucial observables for the reconstruction of side-dependent charge migration is the relative intensity of the even harmonics. Our reconstruction procedure additionally includes the sign-dependence of the transition dipole moment on the molecular orientation (SM section 2.2). The temporal uncertainty in the reconstruction is harmonic-order dependent and amounts to \pm 110 as on average. The temporal accuracy is limited by the uncertainty in the peak intensity needed to connect the electron transit time to the emitted photon energy (22) and the different transit times associated with the electronic states of the cation involved in the dynamics. The shaded area represents the combined error from all experimental uncertainties.

Turning to the results obtained at $1300 \mathrm{~nm}$, we again find strong population transfer in the laser field as shown by the reconstructed populations in Fig. 4c for tunneling via the iodine atom. The fractional ground-state population gradually increases until $1.53 \mathrm{fs}$ and then decreases again. Our reconstruction shows a second minimum of the $\tilde{\mathrm{X}}^{+}$population at $1.71 \mathrm{fs}$ and a subsequent rise for longer delays. For tunneling via the hydrogen atom (Fig. 4d), the behavior is similar with the maximal population shifted to earlier delays. The electron hole densities reconstructed from the populations and relative phases demonstrate the strong laser control over the dynamics. This fact is further illustrated by the supplementary movies (S1 to S4) which 

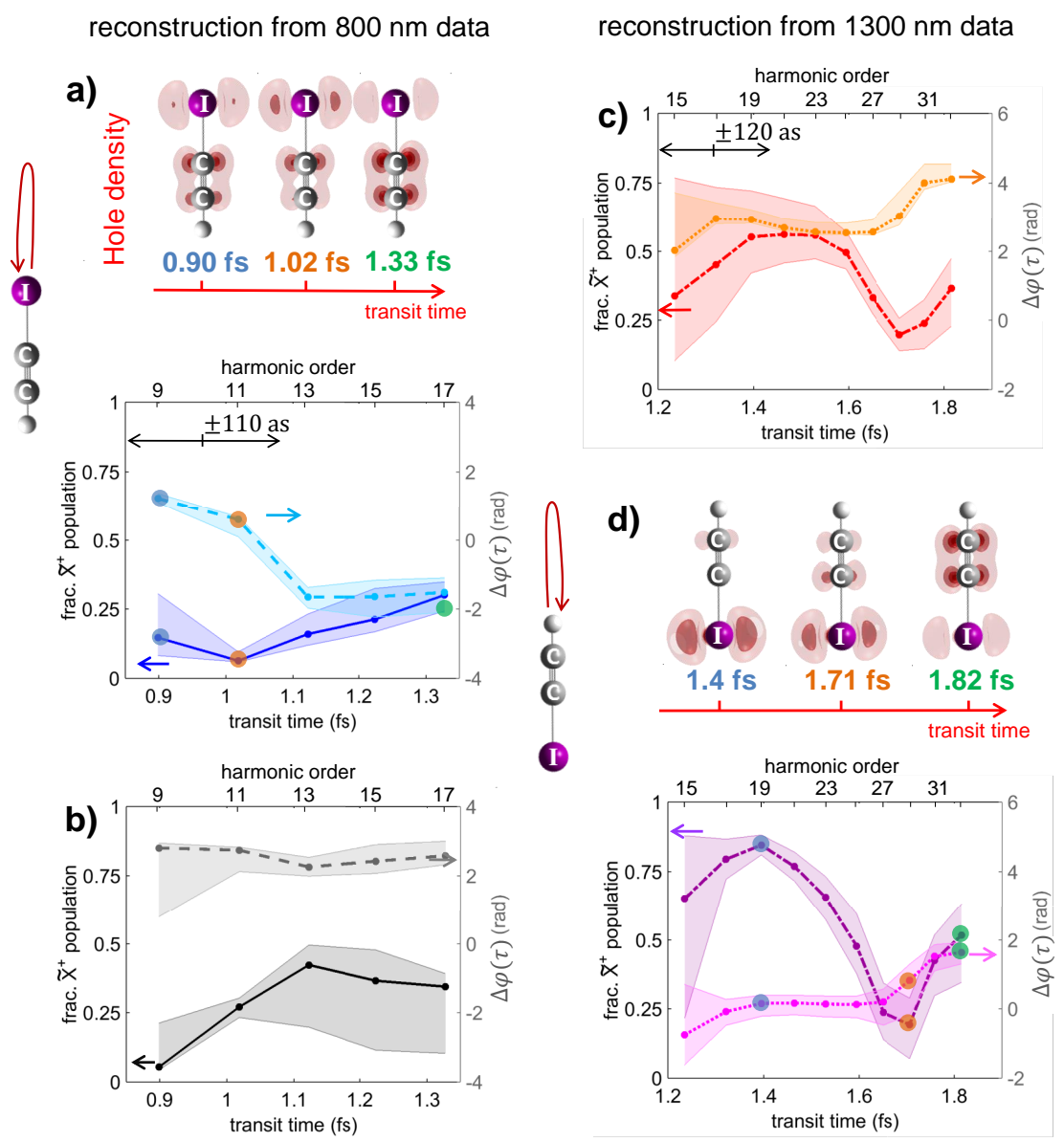

(1)
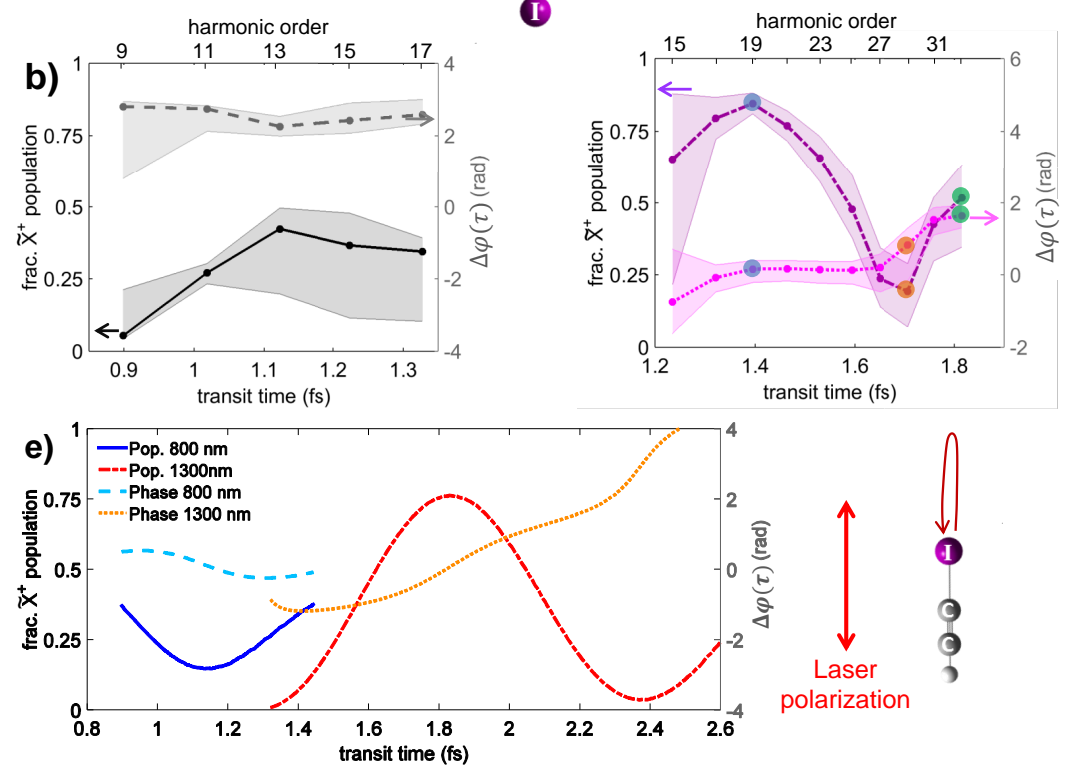

Figure 4: Laser control over attosecond charge migration. (a) Reconstructed hole densities at selected transit times and reconstructed population of the $\tilde{\mathrm{X}}^{+}$state (blue line, the timedependent population of the $\tilde{\mathrm{A}}^{+}$state is given by normalization) and relative phase between the $\tilde{\mathrm{X}}^{+}$and $\tilde{\mathrm{A}}^{+}$states (dashed cyan line) for a driving wavelength of $800 \mathrm{~nm}$ and electron tunneling via the iodine atom. (b) Reconstructed populations and relative phases for tunneling via the hydrogen atom in an 800-nm driving field. (c) Same as (b) for electron tunneling via the iodine atom and $1300 \mathrm{~nm}$ wavelength (d) Same as (a) for tunneling via the hydrogen atom and $1300 \mathrm{~nm}$ wavelength. (e) Independently calculated populations and relative phases for tunneling via the iodine atom using the two experimental wavelengths and peak intensities. Calculations for tunneling via the hydrogen atom and complementary hole densities are given in Fig. S6. 
show very rapid changes in the hole density associated with the reconstructed phase jumps. These results clearly show that both the site of electron tunneling and the laser wavelength offer extensive control over charge migration. They also highlight the highly non-adiabatic nature of the strongly driven electronic dynamics (SM, Fig. S10).

Our experimental reconstruction of the time-dependent populations and phases is supported by independent calculations (Fig. 4e). The population transfer was calculated by solving the time-dependent Schrödinger equation for the two lowest-lying electronic states of the cation with all input taken from high-level ab initio calculations (SM sections 3 and 5) and the relative initial phase $\Delta \varphi$ taken from the experimental reconstruction. We have validated the two-state approach in our reconstruction by comparing time-dependent calculations containing between 2 and 10 electronic states of $\mathrm{HCCI}^{+}$and found that the dynamics are accurately predicted when only the $\tilde{\mathrm{X}}^{+}$and $\tilde{\mathrm{A}}^{+}$states of the cation are included (SM, Fig. S8).

The theoretical population transfer for $800 \mathrm{~nm}$ and tunneling via the I side (blue line in Fig. $4 \mathrm{e})$ correctly predicts the near-complete depletion and repopulation of the ionic ground state, including the associated phase jump. The theoretical depopulation of the ground state is weaker than the reconstructed one (Fig. 4a) and the phase jump is consistently less pronounced. Such jumps are most pronounced for complete de- and repopulations of states (see Fig. S6 which also shows calculations for tunneling via the $\mathrm{H}$ atom). For $1300 \mathrm{~nm}$ the calculations qualitatively agree with the reconstructed dynamics, but the population maximum occurs about 300 as later than in the experimental results. Given the complexity of the non-adiabatic electronic dynamics (Fig. S10), the agreement between experiment (Figs. 4a,c) and calculations (Fig. 4e) is highly encouraging. The non-adiabatic electronic dynamics introduces a strong dependence of the population transfer on the exact parameters of the laser pulse. The remaining discrepancies may result from electron correlation between the continuum wave packet and the bound electrons beyond that taken into account in the present mean-field description of photorecombination.

In conclusion, we have developed a method for reconstructing the quantum dynamics of both laser-controlled and quasi-field-free charge migration from the observables of high-harmonic spectroscopy. Our reconstruction procedure relies on the characterization of high-harmonic 
amplitudes and phases as well as the measurement of even-harmonic emission from oriented molecules. The reconstruction allows for the retrieval of the populations and phases of transiently occupied states of the cation and the characterization of the initial hole created by ionization. We have demonstrated how the interaction with an intense laser field can be either employed to take control over attosecond charge migration or be minimized to reconstruct the quasi-field-free evolution of the hole dynamics. The demonstrated laser control can be further refined by using laser pulses with sub-cycle-controlled waveforms (34).

\section{Acknowledgments}

We thank Manfred Lein for valuable discussions and Franck Lépine, Victor Despré, Pablo Lopez and Ursula Röthlisberger for performing supporting calculations. We gratefully acknowledge funding from an ERC Starting Grant (Project No. 307270-ATTOSCOPE) and the Swiss National Science Foundation via the National Centre of Competence in Research Molecular Ultrafast Science and Technology. FR and BM thank the Fonds National de la Recherche Scientifique of Belgium for its support and the Fonds de la Recherche Fondamentale Collective (grant 2.4545.12). BM thanks the B.A.E.F and W.B.I. for their support. OIT thanks the Ministry of Education and Science of Russia (State assignment No. 3.679.2014/K). LBM acknowledges the VKR Centre of Excellence QUSCOPE and ERC Starting Grants (Project Nos. 277767TDMET). EFP and ADB thank Compute Canada for access to high-performance computers. 


\section{References and Notes}

1. G. S. Engel, et al., Nature 446, 782 (2007).

2. S. M. Falke, et al., Science 344, 1001 (2014).

3. L. Cederbaum, J. Zobeley, Chemical Physics Letters 307, 205 (1999).

4. A. D. Bandrauk, S. Chelkowski, H. S. Nguyen, International Journal of Quantum Chemistry 100, 834 (2004).

5. F. Remacle, R. D. Levine, PNAS 103, 6793 (2006).

6. B. Mignolet, R. D. Levine, F. Remacle, J. Phys. B 47, 124011 (2014).

7. R. Weinkauf, et al., The Journal of Physical Chemistry 100, 18567 (1996).

8. R. Weinkauf, E. W. Schlag, T. J. Martinez, R. D. Levine, J. Phys. Chem. A 101, 7702 (1997).

9. S. R. Leone, et al., Nat. Photon. 8, 162 (2014).

10. M. F. Kling, P. von den Hoff, I. Znakovskaya, R. de Vivie-Riedle, Phys. Chem. Chem. Phys. 15, 9448 (2013).

11. E. Goulielmakis, et al., Nature 466, 739 (2010).

12. A. Fleischer, et al., Phys. Rev. Lett. 107, 113003 (2011).

13. F. Calegari, et al., Science 346, 336 (2014).

14. O. Smirnova, et al., Nature 460, 972 (2009).

15. Y. Mairesse, et al., Phys. Rev. Lett. 104, 213601 (2010).

16. S. Haessler, et al., Nat. Phys. 6, 200 (2010).

17. H. Niikura, et al., Nature 421, 826 (2003). 
18. M. Allan, E. Kloster-Jensen, J. P. Maier, J. Chem. Soc., Faraday Trans. 2 73, 1406 (1977).

19. R. S. Mulliken, The Journal of Chemical Physics 7, 20 (1939).

20. H. Stapelfeldt, T. Seideman, Rev. Mod. Phys. 75, 543 (2003).

21. M. Lein, Phys. Rev. Lett. 94, 053004 (2005).

22. S. Baker, et al., Science 312, 424 (2006).

23. H. J. Wörner, J. B. Bertrand, P. Hockett, P. B. Corkum, D. M. Villeneuve, Phys. Rev. Lett. 104, 233904 (2010).

24. R. Torres, et al., Phys. Rev. A 81, 051802 (2010).

25. C. Vozzi, et al., Nat Phys 7, 822 (2011).

26. S. De, et al., Phys. Rev. Lett. 103, 153002 (2009).

27. X. Zhou, et al., Phys. Rev. Lett. 100, 073902 (2008).

28. A.-T. Le, R. R. Lucchese, S. Tonzani, T. Morishita, C. D. Lin, Phys. Rev. A 80, 013401 (2009).

29. M. Lewenstein, P. Balcou, M. Y. Ivanov, A. L'Huillier, P. Corkum, Phys. Rev. A 49, 2117 (1994).

30. M. V. Frolov, N. L. Manakov, T. S. Sarantseva, A. F. Starace, Journal of Physics B: Atomic, Molecular and Optical Physics 42, 035601 (2009).

31. D. Shafir, et al., Nature 485, 343 (2012).

32. E. F. Penka, E. Couture-Bienvenue, A. D. Bandrauk, Phys. Rev. A 89, 023414 (2014).

33. O. I. Tolstikhin, T. Morishita, L. B. Madsen, Phys. Rev. A 84, 053423 (2011).

34. A. Wirth, et al., Science 334, 195 (2011). 
35. B. Gans, G. Grassi, F. Merkt, The Journal of Physical Chemistry A 117, 9353 (2013).

36. A. Rupenyan, P. M. Kraus, J. Schneider, H. J. Wörner, Phys. Rev. A 87, 033409 (2013).

37. A. Rupenyan, P. M. Kraus, J. Schneider, H. J. Wörner, Phys. Rev. A 87, 031401 (2013).

38. M. J. J. Vrakking, S. Stolte, Chemical Physics Letters 271, 209 (1997).

39. E. Frumker, et al., Physical Review Letters 109, 113901 (2012).

40. P. M. Kraus, A. Rupenyan, H. J. Wörner, Phys. Rev. Lett. 109, 233903 (2012).

41. P. M. Kraus, D. Baykusheva, H. J. Wörner, Phys. Rev. Lett. 113, 023001 (2014).

42. P. M. Kraus, D. Baykusheva, H. J. Wörner, J. Phys. B 47, 124030 (2014).

43. R. Zerne, et al., Phys. Rev. Lett. 79, 1006 (1997).

44. J. B. Bertrand, H. J. Wörner, P. Salières, D. M. Villeneuve, P. B. Corkum, Nat. Phys. 9, 174 (2013).

45. A. Camper, et al., Phys. Rev. A 89, 043843 (2014).

46. M. Ivanov, T. Brabec, N. Burnett, Phys. Rev. A 54, 742 (1996).

47. W. Becker, et al. (Academic Press, 2002), vol. 48 of Advances In Atomic, Molecular, and Optical Physics, pp. $35-98$.

48. A. Becker, F. H. M. Faisal, Journal of Physics B: Atomic, Molecular and Optical Physics 38, R1 (2005).

49. C. F. de Morisson Faria, X. Liu, Journal of Modern Optics 58, 1076 (2011).

50. L. Torlina, O. Smirnova, Phys. Rev. A 86, 043408 (2012).

51. O. Smirnova, M. Ivanov, Attosecond and XUV Physics (Wiley-VCH Verlag GmbH \& Co. KGaA, 2014), pp. 201-256. 
52. T. Morishita, A.-T. Le, Z. Chen, C. D. Lin, Phys. Rev. Lett. 100, 013903 (2008).

53. P. Salières, et al., Science 292, 902 (2001).

54. P. Eckle, et al., Nature Physics 4, 565 (2008).

55. A. S. Landsman, et al., Optica 1, 343 (2014).

56. J. Ortigoso, M. Rodríguez, M. Gupta, B. Friedrich, The Journal of Chemical Physics 110, 3870 (1999).

57. A. Etches, L. B. Madsen, Journal of Physics B: Atomic, Molecular and Optical Physics 43, 155602 (2010).

58. G. L. Yudin, M. Y. Ivanov, Phys. Rev. A 64, 013409 (2001).

59. P. M. Kraus, H. J. Wörner, ChemPhysChem 14, 1445 (2013).

60. A. D. Bandrauk, S. Chelkowski, S. Kawai, H. Lu, Phys. Rev. Lett. 101, 153901 (2008).

61. L. V. Keldysh, Sov. Phys. JETP 20, 1307 (1965).

62. R. R. Lucchese, G. Raseev, V. McKoy, Phys. Rev. A 25, 2572 (1982).

63. R. R. Lucchese, D. Dowek, Attosecond and XUV Physics (Wiley-VCH Verlag GmbH \& Co. KGaA, 2014), pp. 293-320.

64. F. A. Gianturco, R. R. Lucchese, N. Sanna, J. Chem. Phys. 100, 6464 (1994).

65. A. P. P. Natalense, R. R. Lucchese, J. Chem. Phys. 111, 5344 (1999).

66. L. B. Madsen, O. I. Tolstikhin, T. Morishita, Phys. Rev. A 85, 053404 (2012).

67. P. M. Kraus, et al., Nat. Commun. 6, 7039 (2015).

68. L. B. Madsen, F. Jensen, O. I. Tolstikhin, T. Morishita, Phys. Rev. A 87, 013406 (2013). 
69. B. Mignolet, A. Gijsbertsen, M. J. J. Vrakking, R. D. Levine, F. Remacle, Phys. Chem. Chem. Phys. 13, 8331 (2011).

70. V. H. Trinh, V. N. T. Pham, O. I. Tolstikhin, T. Morishita Phys. Rev. A. 91, 063410 (2015).

71. E. Dehghanian, A. D. Bandrauk, G. Lagmago Kamta, The Journal of Chemical Physics 139, (2013).

72. F. Remacle, R. D. Levine, Phys. Rev. A 83, 013411 (2011).

73. R. van Leeuwen, E. J. Baerends, Phys. Rev. A 49, 2421 (1994).

74. H. J. Haink, E. Heilbronner, V. Hornung, E. Kloster-Jensen, Helvetica Chimica Acta 53, 1073 (1970).

75. MOLPRO, version 2010.1, a package of ab initio programs, H.-J. Werner, et al., see http://www.molpro.net.

76. M. J. Frisch, et al., Gaussian, Inc., Wallingford CT, 2009.

77. "Q-Chem: an engine for innovation”, A. I. Krylov, P. M. W. Gill, WIREs Comput Mol Sci 3, 317 (2013)

78. R. Lindh, U. Ryu, and B. Liu, J. Chem. Phys. 95, 5889 (1991)

79. H.-J. Werner and W. Meyer, J. Chem. Phys. 73, 2342 (1980)

80. H.-J. Werner and W. Meyer, J. Chem. Phys. 74, 5794 (1981)

81. H.-J. Werner and P.J. Knowles, J. Chem. Phys. 82, 5053 (1985)

82. P.J. Knowles and H.-J. Werner, Chem. Phys. Lett. 115, 259 (1985)

83. H.-J. Werner, Mol. Phys. 89, 645 (1996)

84. C. Hampel, K. Peterson and H.-J. Werner, Chem. Phys. Lett. 190, 1 (1992)

85. M.J.O. Deegan and P.J. Knowles, Chem. Phys. Lett. 227, 321 (1994) 
86. T.H. Dunning, Jr. J. Chem. Phys. 90, 1007 (1989)

87. K. A. Peterson, B. C. Shepler, D. Figgen, H. Stoll, J. Phys. Chem. A 11013877 (2006).

88. A. Bergner, M. Dolg, W. Kuechle, H. Stoll, H. Preuss, Mol. Phys. 801431 (1993)

89. A. I. Krylov, Annu. Rev. Phys. Chem. 59433 (2008).

90. J. Paldus, in Theory and Applications of Computational Chemistry: The First Forty Years., p. 115, Elsevier, 2005, Amsterdam.

91. R. J. Bartlett, in Theory and Applications of Computational Chemistry: The First Forty Years., p. 1191, Elsevier, 2005, Amsterdam.

92. R. J. Bartlett, M. Musial, Rev. Mod. Phys. 79, 291 (2007).

93. J. C. Saeh, J. F. Stantom, J. Chem. Phys. 1118275 (1999)

94. H. Nakatsuji, in Computational Chemistry - Reviews of Current Trends, Vol. 2, p.62, World Scientific, 1997, Singapore.

95. T. J. Lee, P. R. Taylor, Int. J. Quantum Chem. Quantum Chem. Symp. 23199 (1989)

96. C. L. Janssen, I. M. B. Nielsen, Chem. Phys. Lett. 290423 (1998)

97. T. J. Lee, Chem. Phys. Lett. 372362 (2003)

98. L. S. Cederbaum, W. Domcke, Adv. Chem. Phys. 36205 (1977)

99. W. von Niessen, J. Schirmer, L. Cederbaum, Comp. Rev. Phys. 157 (1984)

100. W. von Niessen, P. Tomassello, J. Schirmer, L. S. Cederbaum, Aust. J. Phys. 39687 (1986)

101. J. V. Ortiz, J. Chem. Phys. 10422 (1996) 
The supplementary material (SM) includes:

Materials and Methods

Supplementary Text

Figures. S1 to S14

Tables S1 to S9

References

Movies S1 to S4 